\title{
A rare cause of inguinal region swelling and pain in women: Hydrocele of the canal of Nuck
}

\author{
Bahjat Barakat, ${ }^{1}$ Massimo Barakat, ${ }^{2}$ Francesco Vasuri, ${ }^{3}$ Raffaele Pezzilli ${ }^{4}$ \\ ${ }^{1}$ Emergency Department, Sant'Orsola Hospital, Bologna; 'Department of Radiology, Sant'Orsola Hospital, \\ Bologna; ${ }^{3}$ Pathology Unit, Sant'Orsola Hospital, Bologna; ${ }^{4}$ Department of Gastroenterology, Sant'Orsola \\ Hospital, Bologna, Italy
}

\begin{abstract}
The most common diagnoses of pelvic/supra-pubic pain are related to uterine, gonadal, renal, and bladder complications. Hydrocele of the canal of Nuck is a rare cause of inguinal swelling in women, which occurs due to a patent processus vaginalis. The canal of Nuck typically closes in infancy, however, in some women, the canal of Nuck remains patent, allowing for the development of canal of Nuck cysts or indirect inguinal hernias. Until now, about 400 cases of this illness have been reported worldwide. Clinically, these cysts are usually fluctuant, painless, or may present locally painful masses in the labia or inguinal regions which are not reducible; we report the case of a young female which was characterized by persistent suprapubic pain in which the diagnosis initially made by ultrasonography was further confirmed by contrast-enhanced computed tomography, surgery and pathological examination.
\end{abstract}

\section{Introduction}

The most common diagnoses of pelvic/supra-pubic pain are related to uterine, gonadal, renal, and bladder complications. Hydrocele of the canal of Nuck is a rare cause of inguinal swelling

Correspondence: Raffaele Pezzilli, Department of Gastroenterology, Sant'Orsola Hospital, Via Massarenti 9, 40138 Bologna, Italy.

Tel./Fax: +39.0512144148

E-mail: raffaele.pezzilli@gmail.com

Key words: Cyst of the canal of Nuck; Female hydrocele; Abdominal ultrasonography; Imaging.

Contributions: the authors contributed equally.

Conflict of interest: the authors declare no potential conflict of interest.

Funding: none.

Ethical statement: the patient gave her informed consent.

Received for publication: 9 May 2019.

Revision received: 29 May 2019.

Accepted for publication: 1 July 2019.

This work is licensed under a Creative Commons Attribution 4.0 License (by-nc 4.0).

C. Copyright: the Author(s), 2019

Licensee PAGEPress, Italy

Emergency Care Journal 2019; 15:8275

doi:10.4081/ecj.2019.8275 in women, which occurs due a patent processus vaginalis. The canal of Nuck typically closes in infancy, however, in some women, the canal of Nuck remains patent, allowing for the development of canal of Nuck cysts or indirect inguinal hernias. Until now, about 400 cases of this illness have been reported worldwide. ${ }^{1}$ Clinically, these cysts are usually fluctuant, painless, or may present locally painful masses in the labia or inguinal regions which are not reducible; ${ }^{2-4}$ thus, we believe it to be of interest to report a case of a young female which was characterized by persistent suprapubic pain.

\section{Case Report}

A 24-year-old Caucasian woman was admitted to the Emergency Department with a six-month' duration right suprapubic pain, which had increased over the last three days. Her past medical history was negative. The patient had normal vital signs and physical investigation revealed the increased intensity of suprapubic pain at palpation, without guarding or rebound tenderness; a soft, cystic, non-compressible, non-fluctuant labial mass, positive at transillumination was found and the overlying skin was normal. She was afebrile and her vital parameters were normal. Laboratory findings revealed an increase in white cell count $(10,730 \mathrm{mmc})$. Renal and hepatic functions were normal, as were C-reactive protein level, serum human chorionic gonadotropin levels and urinalysis. Abdominal ultrasonography (US) revealed a well-defined elongated cystic mass with internal debris and slightly thickened wall, measuring $35 \times 12 \mathrm{~mm}$, located in the right suprapubic region. Compression did not modify the cyst and no vascular signal was demonstrable on a complementary echo-color-Doppler examination (Figure 1). Abdominal contrast-enhanced computed tomography scan (CT) confirmed the presence in the right inguinal canal of a fluid-filled elongated cyst with a slightly thickened wall, which extended from the peritoneal cavity to the homolateral labium majus. Increased parietal uptake of contrast medium was also present, indicating inflammation (Figures 2 and 3). Surgery was required for the persistent pain and after a right skin crease incision, high ligation of the cyst and hydrocelectomy was performed; in addition, the round ligament was excised. At gross pathological examination, the cyst showed a thick wall and an irregular surface, with hemorrhagic areas (Figure 4A). Histologically, the cyst showed a single layer of cuboidal or flattened mesothelial cells strongly positive at immunohistochemistry for calretinin and widespectrum cytokeratins in a highly cellulated fibrous stroma rich in inflammatory cells (Figure 4B-D). The pathological picture was consistent with the diagnosis of hydrocele of the canal of Nuck. ${ }^{5}$ Her postoperative course was uneventful, she was discharged from the hospital and then she went home 3 days after surgery. The patient is doing well at a two-month follow-up with no swelling or recurrence on the operated side. 


\section{Discussion}

First described in 1691 by Anton Nuck, a Dutch anatomist, ${ }^{6}$ the canal of Nuck is the processus vaginalis peritonei; in females this is a tubular fold of peritoneum which invaginates into the inguinal canal anterior to the gubernaculum and descending testis, ending in the scrotum in males or the labium major in females. The upper part usually closes at or just before birth, and obliteration proceeds gradually in a downward direction. Whereas in the male the scrotal section remains patent, in the female, the entire processus vaginalis normally becomes obliterated. When obliteration does not occur, fluid may become encysted within a remnant of the canal of Nuck not communicating with the peritoneal cavity. ${ }^{7}$ Three types of hydrocele of the canal of Nuck are recognized: type 1 is characterized by a unilocular encysted hydrocele which is not communicated with the peritoneal cavity and is the most common finding in clinical practice; type 2 is characterized by a persistent communication between the hydrocele and the peritoneal cavity, as in our case; type 3, which is rarely found, is where the internal inguinal ring is compressed by a large hydrocele and appears as an hourglass. ${ }^{8}$ The cyst formation is probably due to an imbalance of the secretion and absorption of the secretory membrane lining the processus vaginalis; trauma or infection may sometimes cause disruption of lymphatic drainage. ${ }^{2}$

Many physicians are unaware of this malformation ${ }^{9-11}$ and there are usually no acute abdominal symptoms as in the case of an incarcerated inguinal hernia, for which it is often mistaken. Clinical diagnosis may be difficult in one third of patients due to the presence of a concomitant inguinal hernia. ${ }^{2}$ As in our case, even if the diagnosis is easy using an ultrasonographic diagnostic approach, ${ }^{2}$ the imaging technique that is usually used to confirm the US diagnosis is magnetic resonance (MRI). ${ }^{12}$ Unfortunately, in our case, MRI was unavailable for emergency situations and CT was carried out giving the same results as MRI. ${ }^{7}$ However, it has recently been reported that CT may be useful in assessing a proper diagnosis as it shows a thin-walled cystic mass in the inguinal canal with an intra-abdominal extension of the canal of Nuck. ${ }^{13}$ The differential diagnosis of a cystic mass in the female groin region includes round ligament cysts, varicosities of the round ligament, inguinal herniation of the ovary, cystic lymphangiomas, epidermal inclusion cysts, abscesses, and pseudoaneurysms. ${ }^{14}$ With regard to complications, an abscess formation may very rarely result in associated signs and symptoms including fever and increase in severity of pain. The final diagnosis is made during surgery and confirmed by pathological examination. ${ }^{15}$ The pathological examination usually shows the cyst wall partially lined by a stratified squamous, transitional and columnar epithelium and a moderate to heavy active inflammatory component causing erosion and abscess formation may be present. ${ }^{16}$

\section{Conclusions}

We have reported a rare case of female hydrocele; the diagnosis was initially made by US and confirmed by CT, surgical exploration and pathological examination.

Emergency Department physicians should be aware of the presence of cysts of the canal of Nuck as ultrasound diagnosis can avoid further investigations such as CT and MRI and patients can be referred to appropriate treatment.

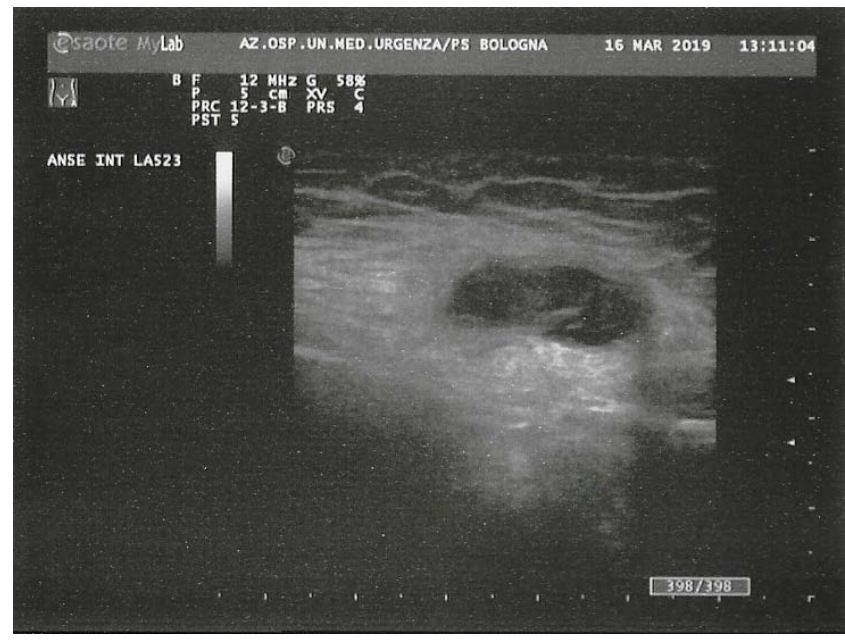

Figure 1. Abdominal ultrasography showing a well-defined ovalar cystic lesion with internal debris and a slightly thickened wall localized in the right suprapubic region.

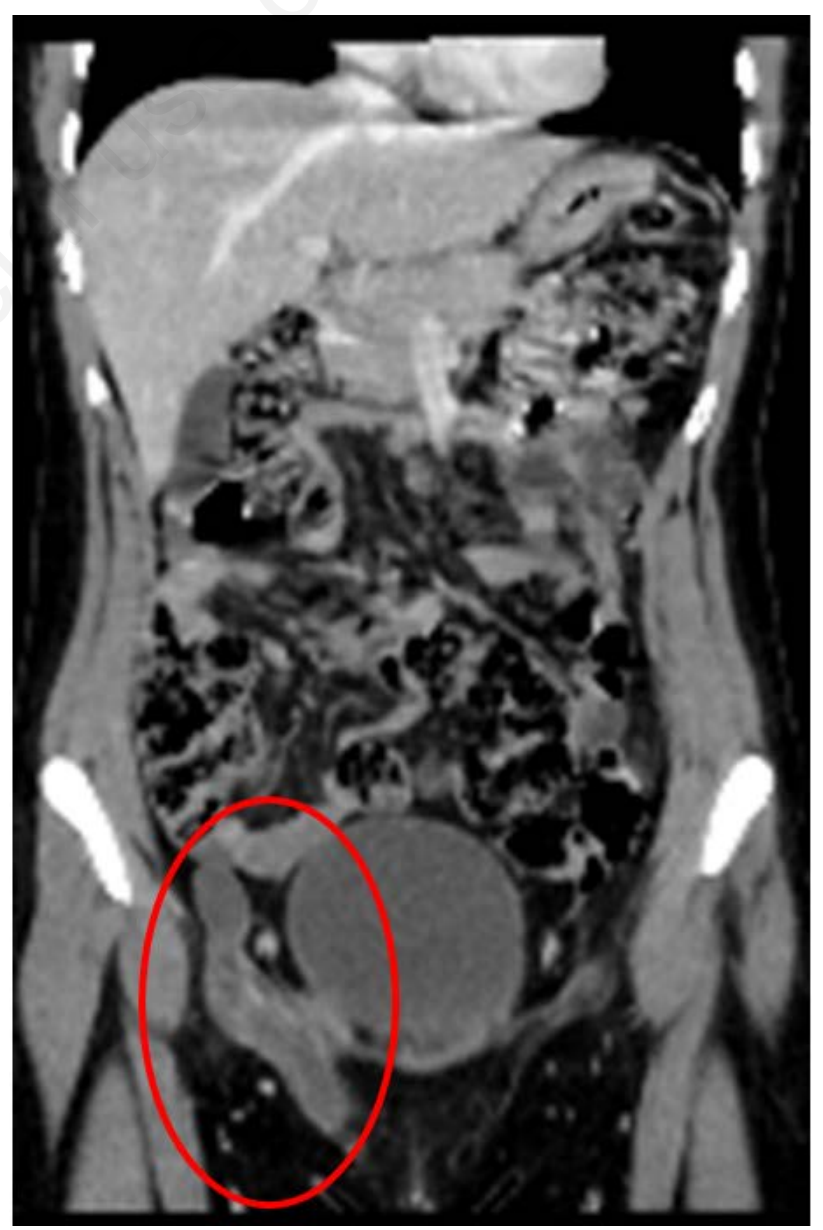

Figure 2. A coronal abdominal contrast-enhanced computed tomography scan showing a fluid-filled elongated cyst with (red circle) a slightly thickened wall in the right inguinal canal; the proximal part of the lesion extends into the peritoneal cavity, while the distal part extends towards the right labium majus. Increased parietal uptake of contrast medium is also present, indicating inflammation. 


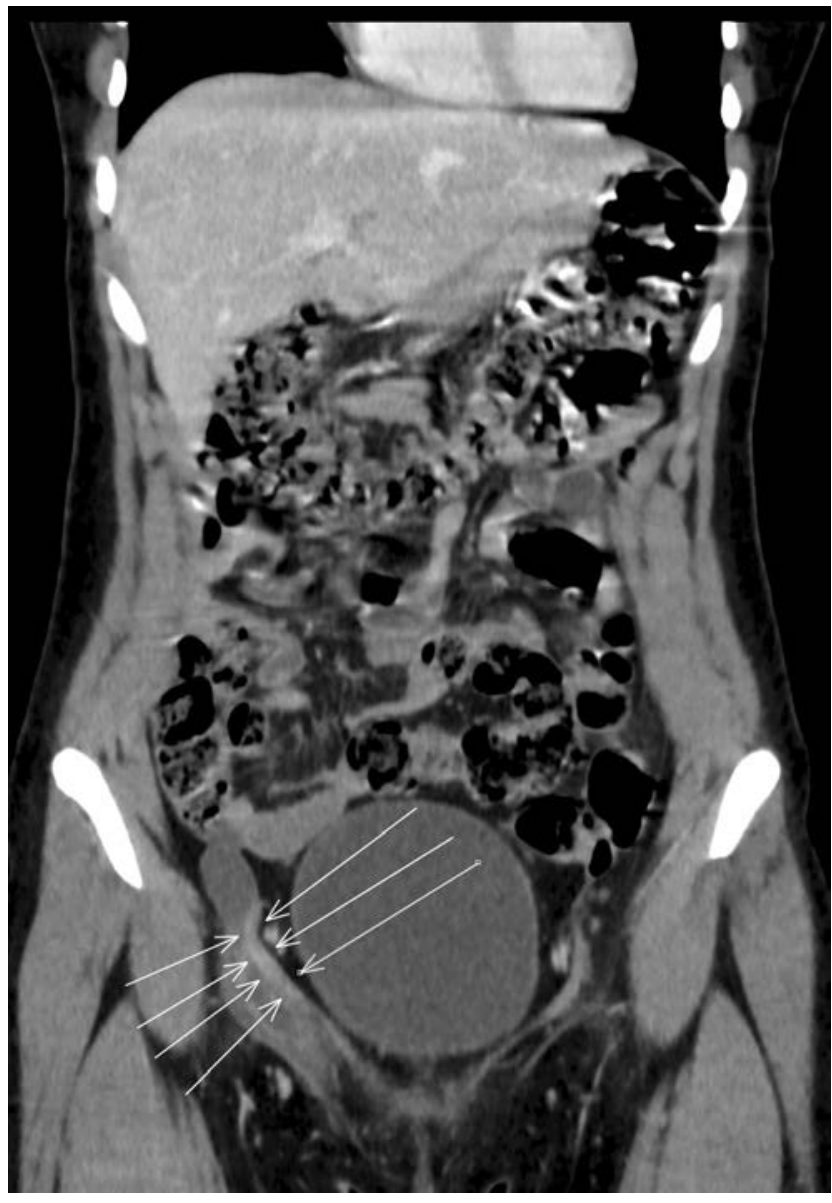

Figure 3. Abdominal contrast-enhanced computed tomography scan showing a well-defined cystic mass in the right inguinal canal and its relation with the homolateral round ligament of the uterus (arrows).

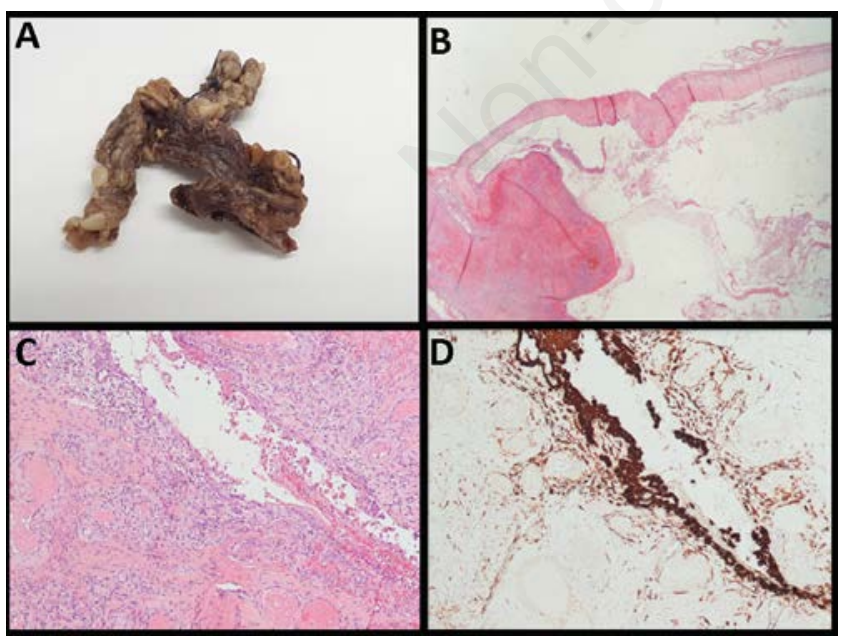

Figure 4. (A) Pathological specimen showing a thick irregular cyst wall. (B, C) At histology, the cyst was lined by a single layer of cuboidal or flattened mesothelial cells in a cellulated stroma (hematoxylin-eosin, $4 \times$ and $20 \times$ magnification). (D) Immunoreactivity for calretinin confirmed the mesothelial nature of the surface cells (20x magnification).

\section{References}

1. Ozel A, Kirdar O, Halefoglu AM, et al. Cysts of the canal of Nuck: ultrasound and magnetic resonance imaging findings. J Ultrasound 2009;12:125-7.

2. Stickel WH, Manner M. Female hydrocele (cyst of the canal of Nuck): sonographic appearance of a rare and little-known disorder. J Ultrasound Med 2004;23:429-32.

3. Yigit H, Tuncbilek I, Fitoz S, et al. Cyst of the canal of Nuck with demonstration of the proximal canal: the role of the compression technique in sonographic diagnosis. J Ultrasound Med 2006;25:123-5.

4. Lai I, Page A, Hamidinia F, Rahmani R. Cysts of the canal of Nuck: A rare sonographic diagnosis. J Clin Ultrasound 2017;45:175-8

5. Kim KS, Choi JH, Kim HM, et al. Hydrocele of the canal of Nuck in a female adult. Arch Plast Surg 2016;43:476-8.

6. Kaeser MA, Haun DW, Cho JCS, Kettner NW. Hydrocele in the canal of Nuck. J Med Ultrasound 2011;19:138-40.

7. Shadbolt CL, Heinze SB, Dietrich RB. Imaging of groin masses: inguinal anatomy and pathologic conditions revisited. Radiographics 2001;21:S261-71.

8. Zvizdic Z, Milisic E, Chikha A, et al. Encysted hydrocele of the canal of Nuck in an 11-month-old child with a past history of duodenal atresia and Arnold-Chiari malformation: A case report. Medicine (Baltimore) 2019;98:e14232.

9. Ihekwaba FN. Hydrocele in the female. J R Coll Surg Edinb 1981;26:91-3.

10. Block RE. Hydrocele of the canal of Nuck: a report of five cases. Obstet Gynecol 1995;45:464-6.

11. Schneider CA, Festa S, Spillert CR, et al. Hydrocele of the canal of Nuck. N J Med 1994;91:37-8.

12. Safak AA, Erdogmus B, Yazici B, Gokgoz AT. Hydrocele of the canal of Nuck: sonographic and MRI appearances. J Clin Ultrasound 2007;35:531-2.

13. Acu L, Acu B. Cyst of the canal of Nuck mimicking inguinal hernia: A case report. OMICS J Radiol 2017;6:251.

14. Oh SN, Jung SE, Rha SE, et al. Sonography of various cystic masses of the female groin. J Ultrasound Med 2007;26:173542.

15. De Meulder F, Wojciechowski M, Hubens G, Ramet J. Female hydrocele of the canal of Nuck: a case report. Eur J Pediatr 2006;165:193-4.

16. Benali F, Gooszen AD, Wetzels C, Piek JMJ. Cyst of Nuck: the importance of histopathological evaluation. Obstet Gynecol Int J 2016;5:00152. 